\title{
IMPORTANCIA DE LOS CUIDADOS EN SITUACIONES COMPLEJAS. UN CASO CLÍNICO Y SU EVOLUCIÓN.
}

\author{
MACIÁ SOLER, A.
}

HERNANDEZ BERMUDEZ, PEDRO J.

Enfermeros clínicos. Servicio de Nefrología. Agudos. Hospital General Universitario de Alicante.

E-mail: antonia macia@hotmail.com 


\section{RESUMEN}

Los cuidados de Enfermería en las unidades de agudos $_{1}$ de Nefrología, presentan una elevada complejidad que requiere destrezas y habilidades especializadas. En el caso del control y seguimiento de pacientes con insuficiencia renal aguda (IRA) complicada, la práctica basada en la evidencia adquiere especial importancia por la toma de decisiones que determinan el pronóstico del paciente.

Se presenta un caso clínico de un paciente que, en el transcurso de una Insuficiencia Renal Aguda (IRA: N.T.A. -N.TI.A.)*, Se diagnostica un mieloma múltiple, donde la actuación del equipo multidisciplinar adquiere especial relevancia por las características del paciente. Se trata de un caso clínico de interés para su estudio y análisis.

Palabras clave:

Insuficiencia renal aguda, mieloma, plan de cuidados.

\section{INTRODUCCIÓN}

Se trata de una persona varón de 47 años que acude al Servicio de Urgencias del Hospital de San Juan por empeoramiento del estado general y fiebre persistente tras ser tratado con levofloxacino por una neumonía causada por Legionella. Durante su ingreso se le diagnostica $\operatorname{IRA}_{1}$ y es trasladado al Hospital General de Alicante (Hospital de referencia del Departamento de salud en Nefrología) e ingresado en la Unidad de Agudos de Nefrología.

En la unidad de agudos de Nefrología, se confirma diagnóstico de Insuficiencia Renal Aguda (IRA) oligoanúrica pre-renal (N.TI.A-N.T.A.), deshidratación y neumonía L.I.D. Durante el periodo de hospitalización para su estudio se diagnostica un Mieloma Múltiple (MM) como causa de la IRA.

En el $50 \%$ de personas que padecen mieloma múltiple se observa insuficiencia renal en el momento del diagnóstico y esta enfermedad representa la segunda causa más común de muerte después de las infecciones 
sistémicas. Es frecuente que presenten inicialmente manifestaciones clínicas renales, y en el transcurso de la evaluación de la enfermedad renal se descubre la presencia de una enfermedad linfoproliferativa ${ }_{2}$. En consecuencia, la planificación de cuidados durante la hospitalización por IRA requiere la consulta de diferentes guías para la práctica y evaluación continua de los resultados de salud del paciente y aplicar procedimientos y técnicas actualizadas.

Se presenta un plan de cuidados, diseñado por la Enfermera responsable del paciente junto con el equipo de cuidados. Para el registro de actividad se utilizan herramientas de las vías clínicas, matriz temporal y registro de verificación y variaciones, con objeto de tratar de sistematizar el proceso asistencial, para garantizar la planificación de actividades, su cumplimiento y evaluación.

Las técnicas y procedimientos se extraen de las guías y manuales disponibles en la Unidad de agudos del Hospital General de Alicante actualizadas con las editadas por la S.E.N.(Sociedad Española de Nefrología) 6 y se explican así como los conceptos clave en anexo.

El proceso de cuidados se valora y evalúa de acuerdo al modelo de patrones funcionales de Gordon:

> Patrón 1 - Percepción / manejo de la salud

$>$ Patrón 2 - Nutricional / metabólico

$>$ Patrón 3 - Eliminación

$>$ Patrón 4 - Actividad / ejercicio

> Patrón 5 - Sueño / descanso

$>$ Patrón 6 - Cognitivo / percetual

$>$ Patrón 7 - Autopercepción / autoconcepto

$>$ Patrón 8 - Rol / relaciones

> Patrón 9 - Sexualidad-reproducción

> Patrón 10 - Adaptación / tolerancia al estrés 
El plan de cuidados tiene dos fases:

- Cuidados en la fase aguda $_{1}$

- Cuidados en hospitalización convencional.

\section{Cuidados en la fase aguda: Descripción del procedimiento de actuación. Registro cuidados.}

Los cuidados en la fase aguda se realizan en la unidad de agudos de Nefrología, donde el ratio enfermera paciente es de 1/3

Los cuidados en agudos comprenden el espacio de tiempo entre el ingreso y el traslado a hospitalización convencional.

La duración de esta fase, varía de acuerdo a la evolución de cada persona y en consecuencia durante este periodo adquiere especial importancia la ausencia de complicaciones derivadas de la hospitalización donde Enfermería tiene capacidad de toma de decisiones.

Para registrar el control y seguimiento se utilizan los registros propios del hospital estructurados de manera que se puedan sistematizar las actividades y observar en sucesivas situaciones similares con objeto de valorar una posible estandarización. El estado del paciente al ingreso en la unidad de agudos y valoración de Enfermería se resumen en la Fig.1.

Signos y síntomas: Fiebre, diarrea, vómitos y oligoanuria de 3 días de evolución.

Juicio clínico: Deterioro de la función renal, IRA ${ }_{1}$ oligoanuria Pre-renal (N.TI.A - N.T.A.), Deshidratación. Neumonía L.I.D.

Valoración equipo clínico:

La oligoanuria es el factor de riesgo vital más importante y la eficacia de la reposición de líquidos intravenosos una prioridad. La composición y velocidad de infusión se ajustará a la evolución del paciente.

Patrones funcionales alterados al ingreso son:

Nutricional/metabolico

Eliminación

El resto de patrones funcionales al ingreso no se evalúan por la gravedad del cuadro agudo.

Se registra al inicio el riesgo de ulceras por presión según la escala Braden y de caídas según la hoja de ingreso de la unidad y del Hospital puntuando en ambas cero por lo que no se recoge la información en las matrices de registro de cuidados.

La actuación de enfermería se dirige hacia los diagnósticos de diarrea,fiebre y desequilibrio de líquidos, priorizando el control de las constantes y reposiciones de líquidos, prestando especial atención a la fluidoterapia y balance hidro-electrolítico.

Fig.1. Estado del paciente al ingreso en la Unidad de agudos de Nefrología 


\section{Planificación de Cuidados:}

Objetivos inmediatos:

- Controlar y mantener las constantes vitales

- Controlar y normalizar el balance hidroelectrolíticos

- Suplir el déficit de autonomía para realizar las actividades básica de la vida diaria

- Evaluación y tratamiento de la ansiedad

\section{Actividades:}

Se recogen en las matrices temporales que se detallan a continuación:

\begin{tabular}{|c|c|c|c|c|c|c|}
\hline Actividades & Día 1 & Evaluación & Día 2 & Evaluación & Día 3 & Evaluación \\
\hline $\begin{array}{l}\text { Determinaciones } \\
\text { Pruebas }\end{array}$ & $\begin{array}{l}\text { Extracción muestras } \\
\text { de sangre. } \\
\text { Recogida de orina }{ }_{2} \text {. } \\
\begin{array}{l}\text { Determinaciones } \\
\text { generales } \\
\text { específicas }\end{array}\end{array}$ & $\begin{array}{l}\text { Deterioro de la } \\
\text { función renal }{ }_{2}\end{array}$ & 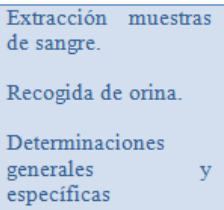 & $\begin{array}{l}\text { Persiste el deterioro } \\
\text { de la función renal }\end{array}$ & $\begin{array}{l}\text { Extracción muestras } \\
\text { de sangre. } \\
\text { Recogida de orina. } \\
\text { Determinaciones } \\
\text { generales y específicas }\end{array}$ & $\begin{array}{l}\text { Persiste el deterioro de la } \\
\text { función renal }\end{array}$ \\
\hline $\begin{array}{l}\text { Administración } \\
\text { fármacos }\end{array}$ & $\begin{array}{l}\text { Fluidos I.V. } \\
\text { Hidratación } \\
\text { Antibióticos I.V. } \\
\text { Antipiréticos. } \\
\text { Antieméticos. } \\
\text { Diuréticos. }\end{array}$ & $\begin{array}{l}\text { Febrícula } \\
\begin{array}{l}\text { Buen manejo de } \\
\text { fluidoterapia. }\end{array} \\
\begin{array}{l}\text { Ausencia de } \\
\text { vómitos }\end{array}\end{array}$ & $\begin{array}{l}\text { Antibioterapia } \\
\text { Fluidoterapia } \\
\text { Antipiréticos. } \\
\text { Diuréticos. }\end{array}$ & $\begin{array}{l}\text { Afebril } \\
\begin{array}{l}\text { Ausencia de } \\
\text { vómitos. } \\
\text { Buen manejo de } \\
\text { fluidoterapia. }\end{array}\end{array}$ & $\begin{array}{l}\text { Antibioterapia } \\
\text { Fluidoterapia. } \\
\text { Diuréticos. } \\
\text { Hipotensores }\end{array}$ & $\begin{array}{l}\text { Afebril. } \\
\text { No vómitos } \\
\text { Buen manejo de los fluidos }\end{array}$ \\
\hline $\begin{array}{l}\text { Patrones } \\
\text { funcionales: } \\
\text { Evaluación } \\
\text { diaria }\end{array}$ & $\begin{array}{l}\text { Nutrición: } \\
\text { Dieta absoluta } \\
\text { Actividad: } \\
\text { Higiene completa en } \\
\text { cama. Dependiente. } \\
\text { Eliminación: } \\
\text { anuria }\end{array}$ & $\begin{array}{l}\text { No vómitos } \\
\text { Autonomía parcial } \\
\text { No diarrea. } \\
\text { Piel hidratada }\end{array}$ & $\begin{array}{l}\text { Nutrición: } \\
\text { Inicia ingesta de } \\
\text { liquidos } \\
\text { Actividad: } \\
\text { Higiene parcial en } \\
\text { lavabo con ayuda. } \\
\text { Rol/relaciones } \\
\text { Eliminación: } \\
\text { Anuria } \\
\text { Deposición en w.c. } \\
\text { No diarrea }\end{array}$ & $\begin{array}{l}\text { Dieta liquida bien } \\
\text { tolerada } \\
\text { Autonomía parcial } \\
\text { por ser portador de } \\
\text { vias invasivas. } \\
\begin{array}{l}\text { Recibe visitas } \\
\text { familiares }\end{array}\end{array}$ & $\begin{array}{l}\text { Nutrición: } \\
\text { Progresa a dieta } \\
\text { blanda. } \\
\text { Actividad: } \\
\text { Camina con ayuda al } \\
\text { lavabo y w.c. } \\
\text { Eliminación: } \\
\text { Anuria } \\
\text { No diarrea }\end{array}$ & $\begin{array}{l}\text { Dieta bien tolerada. } \\
\text { No diarrea. } \\
\text { Autonomia parcial. } \\
\text { Se levanta con ayuda al sillón } \\
\text { y al lavabo. }\end{array}$ \\
\hline $\begin{array}{l}\text { Balance } \\
\text { hidroelctrolítico }\end{array}$ & $\begin{array}{l}\text { Control y registro } \\
\text { entradas-salidas } \\
\text {-Peso diario } \\
\text {-Balance diario }\end{array}$ & $\begin{array}{l}\text { Balance positivo } \\
\text { Anuria } \\
\text { Aumento de peso }\end{array}$ & $\begin{array}{l}\text { Control y registro de } \\
\text { entradas y salidas } \\
\text { Peso y balance }\end{array}$ & $\begin{array}{l}\text { Balance positivo } \\
\text { Anuria } \\
\text { Aumento de peso }\end{array}$ & $\begin{array}{l}\text { Control y registro de } \\
\text { entradas y salidas } \\
\text { Peso y balance }\end{array}$ & $\begin{array}{l}\text { Balance positivo. } \\
\text { Persiste la anuria. } \\
\text { Aumento de peso }\end{array}$ \\
\hline Constantes & $\begin{array}{l}\text { Saturación de } \\
\text { oxigeno. } \\
\text { T.A. y T } \\
\text { Frecuencia cardíaca } \\
\text { Monitorización }\end{array}$ & $\begin{array}{l}\text { Mejora la saturación } \\
\text { y se mantienen las } \\
\text { constantes. }\end{array}$ & $\begin{array}{l}\text { Toma y registro } \\
\text { Monitorización }\end{array}$ & $\begin{array}{l}\text { Mejora la } \\
\text { saturación. } \\
\text { Aparece } \\
\text { hipertensión. }\end{array}$ & $\begin{array}{l}\text { Toma y registro } \\
\text { Monitorización }\end{array}$ & $\begin{array}{l}\text { Saturaciones alrededor del } \\
98 \% \\
\text { Sigue hipertenso } \\
\text { Frecuencia cardiaca dentro de } \\
\text { la normalidad }\end{array}$ \\
\hline
\end{tabular}




\begin{tabular}{|c|c|c|c|c|}
\hline Actividades & Dia 4 & Evaluación & Dia 5 & Evaluación \\
\hline $\begin{array}{l}\text { Pruebas } \\
\text { Determinaciones }\end{array}$ & $\begin{array}{l}\text { Mañana Biopsia renal }{ }_{5}: \text { Preparación } \\
\text { Se inserta C.V.C. no tunelizado en } \\
\text { femoral } 4 \text {. } \\
\text { Hoy } 1^{\text {a }}{ }^{-D_{-2}} \text { con ultrafiltación } \\
\text { Transfusión }\end{array}$ & $\begin{array}{l}\text { H.D. Bien tolerada. } \\
\text { No sangrado zona cateter. } \\
\text { No aparecen reacciones adversas a la } \\
\text { transfusión }\end{array}$ & $\begin{array}{l}\text { Realización de la Biopsia } \\
\text { Cuidados Post-Biopsia }\end{array}$ & $\begin{array}{l}\text { No signos ni sintomas de } \\
\text { sangrado }_{5} \text {. }\end{array}$ \\
\hline $\begin{array}{l}\text { Administración } \\
\text { fármacos }\end{array}$ & $\begin{array}{l}\text { Pre-H.D } \\
\text { Post-HD } \\
\text { Oral-I.V. }\end{array}$ & $\begin{array}{l}\text { Se administran antibioticos post-H.D. } \\
\text { Dolor por la inserción del } \\
\text { C.V.C.:Analgesia I.V. }\end{array}$ & $\mathrm{TT}^{\circ}$ igual & $\mathrm{TT}^{\circ}$ bien tolerado \\
\hline $\begin{array}{l}\text { Patrones } \\
\text { funcionales: } \\
\text { Evaluación } \\
\text { diaria }\end{array}$ & $\begin{array}{l}\text { Actividad } \\
\text { Eliminacion } \\
\text { Percepción/manejo de salud } \\
\text { Cognitivo/perceptual }\end{array}$ & $\begin{array}{l}\text { Se levanta con ayuda } \\
\text { Pasea limitado por el cateter femoral } \\
\text { Anuria } \\
\text { Solicita información de su proceso } \\
\text { Dolor }\end{array}$ & $\begin{array}{l}\text { Actividad } \\
\text { Reposo absoluto } \\
\text { Eliminación } \\
\text { Percepción/manejo de salud } \\
\text { Cognitivo/perceptual }\end{array}$ & $\begin{array}{l}\text { Higiene en cama } \\
\text { Come sentado } \\
\text { Anuria } \\
\text { Conoce las causaa y evolucion } \\
\text { de su enfermedad } \\
\text { No dolor }\end{array}$ \\
\hline Balance & $\begin{array}{l}\text { Se añade la ultrafiltración a las salidas del } \\
\text { balance }\end{array}$ & Se aproxima a cero & Control y registro entradas-salidas & Balances equilibrados \\
\hline Constantes & $\begin{array}{l}\text { Temperatura. } \\
\text { Monitorización } \\
\text { T.A. F.C. Sat } \mathrm{O}_{2}\end{array}$ & $\begin{array}{l}\text { Afebril } \\
\text { Sin cambios en registros } \\
\text { hipertenso }\end{array}$ & $\begin{array}{l}\text { Temperatura. } \\
\text { Monitorización } \\
\text { T.A. F.C. Sat } \mathrm{O}_{2}\end{array}$ & $\begin{array}{l}\text { Afebril } \\
\text { Sin cambios en registros } \\
\text { hipertenso }\end{array}$ \\
\hline
\end{tabular}

Matriz temporal. Registro de la fase aguda 2

\begin{tabular}{|c|c|c|c|c|c|c|}
\hline Actividades & Día 6 & Evaluación & Día 7 & Evaluación & Día 8 & Evaluación \\
\hline Pruebas & H.D. & H.D.Bien tolerada & H.D. & H.D.Bien tolerada & H.D. & H.D.Bien tolerada \\
\hline Determinaciones & A.S. & $\begin{array}{l}\text { Obtención de } \\
\text { muestras en H.D. }\end{array}$ & A.S. & A.S. En H.D. & A.S. & A.S. En H.D. \\
\hline $\begin{array}{l}\text { Administración } \\
\text { fármacos }\end{array}$ & $\begin{array}{l}\text { Oral } \\
\text { I.V. } \\
\text { Pre-H.D. } \\
\text { Post-H.D. }\end{array}$ & $\begin{array}{l}\text { Se coordina } \\
\text { donde medicación } \\
\text { i.v. } \\
\text { con las sesiones } \\
\text { de H.D. }\end{array}$ & $\begin{array}{l}\text { Se suspenden los fluidos. } \\
\text { TT Oral }^{\circ} \text { Oral } \\
\text { TT }^{\circ} \text { I.V. en H.D. }\end{array}$ & $\begin{array}{l}\text { Se coordina donde } \\
\text { TT }^{\circ} \\
\text { Bien tolerado }\end{array}$ & $\begin{array}{l}\text { Se suspende medicación } \\
\text { I.V. } \\
\text { Antibióticos I.V.en H.D. } \\
\text { Se retira via periférica }\end{array}$ & $\begin{array}{l}\text { Buena tolerancia } \\
\text { al TT }\end{array}$ \\
\hline $\begin{array}{l}\text { Patrones } \\
\text { funcionales: } \\
\text { Evaluación }\end{array}$ & \multirow[t]{2}{*}{$\begin{array}{l}\text { Actividad } \\
\text { Eliminación } \\
\text { Percepción } \\
\text { /manejo de salud }\end{array}$} & $\begin{array}{l}\text { Autonomo } \\
\text { Aumento de } \\
\text { diuresis }\end{array}$ & \multirow[t]{2}{*}{$\begin{array}{l}\text { Eliminación } \\
\text { Percepción } \\
\text { /manejo de salud }\end{array}$} & \multirow[t]{2}{*}{$\begin{array}{l}\text { Oligoanuria en remision } \\
\text { Manifiesta satisfaccion con la } \\
\text { información recibida }\end{array}$} & $\begin{array}{l}\text { Eliminacion } \\
\text { Percepción } \\
\text { /manejo de salud }\end{array}$ & $\begin{array}{l}\text { Se retira sonda } \\
\text { vesical } \\
\text { Control diuresis } \\
\text { cada } 24 \mathrm{hrs}\end{array}$ \\
\hline diaria & & $\begin{array}{l}\text { Solicita } \\
\text { información de su } \\
\text { proceso }\end{array}$ & & & & $\begin{array}{l}\text { Informamos al } \\
\text { paciente sobre la } \\
\text { recogida de orina }\end{array}$ \\
\hline \multirow[t]{3}{*}{ Balance } & \multirow[t]{3}{*}{$\begin{array}{l}\text { Control y } \\
\text { registro entradas } \\
\text { y salidas }\end{array}$} & $\begin{array}{l}\text { Balance } \\
\text { equilibrado }\end{array}$ & $\begin{array}{l}\text { Control y registro entradas } \\
\text { y salidas }\end{array}$ & $\begin{array}{l}\text { Diuresis dentro de los } \\
\text { parámetros normales. }\end{array}$ & $\begin{array}{l}\text { Control y registro } \\
\text { entradas y salidas }\end{array}$ & $\begin{array}{l}\text { Diuresis } \\
\text { restablecida. }\end{array}$ \\
\hline & & $\begin{array}{l}\text { Disminuye la } \\
\text { UFR en H.D. }\end{array}$ & & $\begin{array}{l}\text { Disminuye la UFR en H.D. } \\
\text { Sigue con balances } \\
\text { equilibrados. }\end{array}$ & & $\begin{array}{l}\text { Disminuye la } \\
\text { UFR en H.D. }\end{array}$ \\
\hline & & & & & & $\begin{array}{l}\text { Balance } \\
\text { eqilibrado }\end{array}$ \\
\hline \multirow[t]{3}{*}{ Constantes } & Temperatura. & Sat. Normal & $\begin{array}{l}\text { Se retira monitor y } \\
\text { saturimetro }\end{array}$ & Hipertenso & T.A. y $T^{a}$ por turno & Afebril \\
\hline & Monitorización & & \multirow[t]{2}{*}{ Control $\mathrm{T}^{\mathrm{a}}$ y $\mathrm{T}$.A. por tumo } & & & $\begin{array}{l}\text { T.A.Controlada } \\
\text { con tendencia a la }\end{array}$ \\
\hline & $\begin{array}{l}\text { T.A. F.C. E.C.G. } \\
\text { Sat } \mathrm{O}_{2}\end{array}$ & & & & & H.T.A. \\
\hline
\end{tabular}

Matriz temporal. Registro de la fase aguda 3 


\section{Registros de verificación y variaciones: Evolución}

Al tercer día de estancia se decide iniciar diálisis ${ }_{2}$ ante la persistencia del deterioro de la función renal que se manifiesta por las alteraciones en las cifras analíticas analítica, anuria, hipertensión, balances positivos y ganancia de peso a pesar de haber mejorado el estado general del paciente y haber obtenido un buen control de las constantes vitales . $_{1}$

Juicio clínico : Neumonia L.I.D. Persiste Oligoanuria + sobrecarga hidrica + N.T.A.- N.TI.A. (etilogía multifactorial:antibioticos para combatir legionella) 1

Se decide iniciar hemodiálisis ( H.D) tras la inserción de cateter femoral temporal.

Se programa biopsia renal.

(Ver en anexo, los puntos de énfasis de la técnicas)

\section{Evaluación de la fase aguda:}

El octavo día el paciente presenta una mejoría del estado general con restauración de la diuresis y normalización de las constante vitales, asi como una buena evolución de sus Patrones Funcionales. Se decide su traslado a planta de hospitalización convencional para continuar tratamiento de la neumonía en remisión y la IRA persistente. Se inicia estudio de pico monoclonal Kappa por hallazgo analítico:Pico Monoclonal a valorar por la unidad de hematología .

Tiene autonomía completa para realizar las necesidades básicas de la vida diaria y buena tolerancia a la medicación y a las diálisis. No presenta problemas para dormir, ni para comer, ni problemas de eliminación. Se le 
enseña a recoger orina cada 24 horas para analítica.

Acepta bien el tratamiento y está bien informado tanto él como su pareja sobre diagnóstico, pronostico y tratamiento de la enfermedad. Los patrones que se evalúan de manera continuada desde el ingreso hasta el alta son: eliminación; percepción/manejo de salud y rol/relaciones.

Sigue con H.D. diarias aunque en ellas se programa cada vez menos ultrafiltración (U.F.R.) por la restauración de la diuresis.

El registro de verificación de actividad es específico de la unidad y no presenta errores de cumplimiento.

\section{Cuidados en hospitalización convencional: Descripción del procedimiento de actuación. Registro cuidados.}

\section{Registro planta-agudos}

En los días sucesivos a la estancia en la unidad de agudos el paciente permanecerá ingresado en planta de Nefrología con modelo de cuidados de hospitalización convencional y acudirá a la Unidad de Agudos exclusivamente a realizarse Hemodialisis.

\section{Planificación de Cuidados:}

Objetivos:

- Contribuir a la restitución de la función renal.

- Coordinar con la Unidad de Agudos las técnicas y tratamientos compartidos.

- Proporcionar la informacion a demanda de cada preparación y realización de los procedimiento y sus posibles cambios de pronostico y tratamiento.

Actividades:

Se recogen en las matrices temporales que se presentan a continuación. 


\begin{tabular}{|c|c|c|c|c|c|c|c|c|}
\hline Actividades & Día 9 & Evaluación & Día 10 & Evaluación & Día 11 & Evaluación & Día 12 & Evaluación \\
\hline Pruebas & $\begin{array}{l}\text { H.D. } \\
\text { A.S. }\end{array}$ & $\begin{array}{l}\text { Hallazgo } \\
\text { pico monoclonal }\end{array}$ & $\begin{array}{l}\text { H.D. dias } \\
\text { alternos }\end{array}$ & $\begin{array}{l}\text { Mejoría de la } \\
\text { función renal }\end{array}$ & A.S. & $\begin{array}{l}\text { Pendiente } \\
\text { Valoración } \\
\text { hematología }\end{array}$ & $\begin{array}{l}\text { H.D. } \\
\text { Aspirado de médula }{ }^{2}\end{array}$ & Precisa analgesia oral. \\
\hline Admon.TT ${ }^{\circ}$ & Oral en planta. & Bien tolerado & Se coordina & Afebril & No & Se reducen & Adon $\mathrm{TT}^{\circ}$ & Sigue con $\mathrm{TT}^{\circ}$ \\
\hline Toma de constantes & $\begin{array}{l}\text { I.V. en H.D. } \\
T^{\mathrm{a}} \text { y T.A. por turno }\end{array}$ & $\begin{array}{l}\text { Afebril. } \\
\text { T.A: } \\
\text { Controlada con } \\
\text { tendencia a la H.T.A }\end{array}$ & $\begin{array}{l}\text { TT }^{\circ} \text { I.V. con } \\
\text { Agudos y Unidosis }\end{array}$ & Normotenso & $\mathrm{HD}$ & los hipotensores & $\begin{array}{l}\text { I.V. } \\
\text { En } \\
\text { Agudos }\end{array}$ & $\begin{array}{l}\text { Antibiótico. } \\
\text { Neumonía } \\
\text { resolución. }\end{array}$ \\
\hline $\begin{array}{l}\text { Patrones } \\
\text { funcionales. } \\
\text { Evaluación diaria }\end{array}$ & $\begin{array}{l}\text { Eliminación } \\
\text { Percepción/ } \\
\text { Manejo de } \\
\text { Salud. } \\
\text { Rol/relaciones }\end{array}$ & $\begin{array}{l}\text { Restauración de la } \\
\text { diuresis. } \\
\text { Mejoría de la función } \\
\text { renal } \\
\text { Esta informado } \\
\text { Siempre acompañado }\end{array}$ & $\begin{array}{l}\text { Percepción/ } \\
\text { Manejo de } \\
\text { Salud. } \\
\text { Rol/relaciones }\end{array}$ & $\begin{array}{l}\text { Refiere información } \\
\text { suficiente. } \\
\text { Acompañado } \\
\text { Siempre por familiar }\end{array}$ & $\begin{array}{l}\text { Percepción/ } \\
\text { Manejo de } \\
\text { Salud } \\
\text { Rol/relaciones }\end{array}$ & $\begin{array}{l}\begin{array}{l}\text { Muestra } \\
\text { indicios } \\
\text { confusión de } \\
\text { negación. }\end{array} \\
\text { Apoyo familiar }\end{array}$ & $\begin{array}{l}\text { Percepción/ } \\
\text { Manejo de } \\
\text { Salud. } \\
\text { Rol/relación } \\
\text { Cognitivo/perceptual }\end{array}$ & $\begin{array}{l}\text { Confuso. } \\
\text { Iritable. } \\
\text { Acompañado. } \\
\text { Apoyo familiar } \\
\text { Dolor }\end{array}$ \\
\hline
\end{tabular}

\begin{tabular}{|c|c|c|c|c|}
\hline Actividades & Día 13 & Evaluación & Día 14 & Evaluación \\
\hline $\begin{array}{l}\text { Pruebas } \\
\text { Analiticas }\end{array}$ & $\begin{array}{l}\text { Extracción de } \\
\text { muestras para } \\
\text { analitica de sangre }\end{array}$ & $\begin{array}{l}\text { Pte. Valoración } \\
\text { por } \\
\text { hematología }\end{array}$ & $\begin{array}{l}\text { H.D. } \\
\text { Recambio cateter femoral } \\
\text { en agudos por mal } \\
\text { funcionamiento. } \\
\text { Refiere dolor } \\
\text { Mañana Biopsia renal }\end{array}$ & $\begin{array}{l}\text { Buen } \\
\text { funcionamiento }\end{array}$ \\
\hline $\begin{array}{l}\text { Administración } \\
\text { medicación }\end{array}$ & $\begin{array}{l}\text { Se asocia } \\
\text { diacepan oral }\end{array}$ & $\begin{array}{l}\text { Descansa } \\
\text { de noche }\end{array}$ & $\begin{array}{l}\text { Se disminuyen } \\
\text { Antibiot.i.v. } \\
\text { Demanda analgesia i.v. } \\
\text { solo en agudos }\end{array}$ & $\begin{array}{l}\text { Buen control } \\
\text { del dolor. } \\
\text { Analgesia }\end{array}$ \\
\hline Constantes & $\begin{array}{l}\mathrm{T}^{\mathrm{a}} \\
\mathrm{T} . \mathrm{A} .\end{array}$ & Ligera H.T.A. & $\begin{array}{l}\mathrm{T}^{\mathrm{a}} \\
\text { T.A. }\end{array}$ & $\begin{array}{l}\text { T.A. dentro } \\
\text { de la normalidad } \\
\text { afebril }\end{array}$ \\
\hline $\begin{array}{l}\text { Patrones } \\
\text { funcionales. } \\
\text { Evaluación diaria }\end{array}$ & $\begin{array}{l}\text { Percepción } \\
\text { /manejo de salud } \\
\text { Sueño/descanso }\end{array}$ & $\begin{array}{l}\text { Ansiedad } \\
\text { Insomnio } \\
\text { Confuso } \\
\text { Descansa } \\
\text { de noche }\end{array}$ & $\begin{array}{l}\text { Percepción } \\
\text { /manejo de salud } \\
\text { Sueño/descanso }\end{array}$ & $\begin{array}{l}\text { Ansiedad } \\
\text { Irritabilidad } \\
\text { Duerme. } \\
\text { Demanda mucha } \\
\text { información. } \\
\text { Buen apoyo familiar }\end{array}$ \\
\hline
\end{tabular}

Matriz temporal. Registro de cuidados generales 2

\begin{tabular}{|c|c|c|c|c|}
\hline Actividad & Día 15 & Evaluación & Día 16 & Evaluación \\
\hline $\begin{array}{l}\text { Pruebas } \\
\text { Analiticas }\end{array}$ & $\begin{array}{l}\text { Nueva biopsia } \\
\text { Mañana } \\
\text { C.V.C.4 } \\
\text { Permcath }\end{array}$ & Sin complicaciones & $\begin{array}{l}\text { Se inserta nuevo } \\
\text { C.V.C. } \\
\text { H.D. } \\
\text { A.S. } \\
\text { Refiere dolor. }\end{array}$ & $\begin{array}{l}\text { Buen } \\
\text { Funcionamiento } \\
\text { del Permcath. } \\
\text { No sangrado. } \\
\text { H.D. bien tolerada }\end{array}$ \\
\hline $\begin{array}{l}\text { Administración } \\
\text { medicación }\end{array}$ & Analgesia oral & $\begin{array}{l}\text { Buen control } \\
\text { Del dolor }\end{array}$ & $\begin{array}{l}\text { Analgesia i.v. en Agudos } \\
\text { oral en planta }\end{array}$ & $\begin{array}{l}\text { Buen control } \\
\text { Del dolor }\end{array}$ \\
\hline Constantes & Control post-biopsia & $\begin{array}{ll}\text { Sin signos } & \mathrm{ni} \\
\text { sintomas } & \text { de } \\
\text { sangrado } & \end{array}$ & $\begin{array}{l}\mathrm{T}^{\mathrm{a}} \\
\text { T.A. }\end{array}$ & $\begin{array}{l}\text { Normotenso. } \\
\text { Afebril }\end{array}$ \\
\hline $\begin{array}{l}\text { Patrones funcionales } \\
\text { Evaluación diaria }\end{array}$ & $\begin{array}{l}\text { Actividad } \\
\text { Percepción } \\
\text { /manejo de salud }\end{array}$ & $\begin{array}{l}\text { Reposo absoluto. } \\
\text { Acompañado por su } \\
\text { mujer dia y noche. }\end{array}$ & $\begin{array}{l}\text { Percepción } \\
\text { /manejo de salud }\end{array}$ & $\begin{array}{l}\text { No ansiedad } \\
\text { ni irritabilidad }\end{array}$ \\
\hline & Sueño/descanso & $\begin{array}{l}\text { Come y duerme } \\
\text { bien. }\end{array}$ & Sueño/descanso & \\
\hline
\end{tabular}

Matriz temporal. Registro de cuidados generales 3 


\begin{tabular}{|c|c|c|c|c|c|c|}
\hline Recien & & Procedimientc & s: Caso clínico & & Revista Cient & tífica de Enfermería \\
\hline Actividades & Díal7 & Evaluación & Díals & Evaluación & Día19 & Evaluacióon \\
\hline $\begin{array}{l}\text { Pruebas } \\
\text { Analiticas }\end{array}$ & $\begin{array}{l}\text { A.S. } \\
\text { Retiramos en } \\
\text { agudos cateter } \\
\text { Femoral. } \\
\text { Vigilancia en planta } \\
\text { del punto de } \\
\text { inserción. }\end{array}$ & $\begin{array}{l}\text { Mejoría de la } \\
\text { función renal. } \\
\text { No sangrado. }\end{array}$ & $\begin{array}{l}\text { H.D. por Permcath. } \\
\text { Próximo dia se } \\
\text { programa última } \\
\text { diálisis }\end{array}$ & $\begin{array}{l}\text { H.D. sin } \\
\text { problemas }\end{array}$ & Ultima H.D. & $\begin{array}{l}\text { H.D. } \\
\text { Sin Problemas. } \\
\text { Función renal restituida. }\end{array}$ \\
\hline $\begin{array}{l}\text { Adon. } \\
\text { medicación }\end{array}$ & $\begin{array}{l}\text { Se prepara nuevo } \\
\text { tratamiento de } \\
\text { hematología }\end{array}$ & Sin problemas & $\begin{array}{l}\text { Quimioterapia } \\
\text { post-H.D. } \\
\text { se administra por } \\
\text { C.V.C. } \\
\text { No se retira } \\
\text { Permcath }\end{array}$ & $\begin{array}{l}\mathrm{TT}^{\circ} \text { bien } \\
\text { tolerado }\end{array}$ & $\begin{array}{l}\text { Quimioterapia } \\
\text { Post-H.D. } \\
\text { por C.V.C. }\end{array}$ & Sin efectos adversos \\
\hline Constantes & $\begin{array}{l}\text { Por turno: } \\
\text { Ta } \\
\text { T.A. }\end{array}$ & $\begin{array}{l}\text { Normotenso } \\
\text { afebril }\end{array}$ & $\begin{array}{l}\text { Por tumo } \\
\mathrm{T}^{\mathrm{a}} \\
\text { T.A. }\end{array}$ & $\begin{array}{l}\text { Normotenso } \\
\text { afebril }\end{array}$ & $\begin{array}{l}\text { Por turno } \\
\mathrm{T}^{\mathrm{a}} \\
\text { T.A. }\end{array}$ & $\begin{array}{l}\text { Normotenso } \\
\text { afebril }\end{array}$ \\
\hline $\begin{array}{l}\text { Patrones } \\
\text { funcionales } \\
\text { Evaluación } \\
\text { diaria }\end{array}$ & $\begin{array}{l}\text { Actividad } \\
\text { Percepción } \\
\text { /manejo de salud } \\
\text { Sueño/descanso }\end{array}$ & $\begin{array}{l}\text { Autonomo } \\
\text { Tranquilo. } \\
\text { Duerme bien } \\
\text { Habla sobre su } \\
\text { enfermedad. }\end{array}$ & $\begin{array}{l}\text { Percepción } \\
\text { /manejo de salud } \\
\text { Sueño/descanso }\end{array}$ & $\begin{array}{l}\text { Animado por la } \\
\text { supresión de las } \\
\text { diálisis. }\end{array}$ & $\begin{array}{l}\text { Actividad } \\
\text { Percepción } \\
\text { /manejo de } \\
\text { salud } \\
\text { Sueño/descanso } \\
\text { Eliminacion }\end{array}$ & $\begin{array}{l}\text { Buen afrontamiento del } \\
\text { diagnostico y } \\
\text { tratamiento }\end{array}$ \\
\hline
\end{tabular}

\section{Evaluación}

Se confirma diagnóstico: IRA compatible con Mieloma Multiple 1,2,3 .

Se inicia tratamiento quimioterápico a administrar en la próxima sesión de H.D. en la unidad de agudos por la Via Central.

Mejoria importante de la funcion renal. Se suspenden las diálisis.

Sigue ingresado en la planta de Nefrologia para control de la función renal con interconsulta a Hematologia para seguimiento y tratamiento del MM.No se retira el cateter permacath por su posible uso para administración de quimioterapia.

La afectación renal en estos pacientes que conlleve a un fracaso renal irreversible requiere una intervención rápida y conjunta de todo el equipo de nefrología que tenga como objetivo la restauracion de la función renal del paciente con mieloma múltiple. 
Es autónomo para todas las actividades, tiene restaurado el patron de eliminación y se mantiene con las constantes en cifras normales. Manifiesta tener información completa sobre su diagnóstico, tratamiento y pronóstico.

\section{Registros y análisis de Enfermería.}

El registro utilizado durante los ocho primeros días en la Unidad de Agudos es una matriz temporal de vía clínica que, se puede aplicar a todas las personas con IRA que ingresen en la Unidad, adaptándolo a las individualidades de cada persona independientemente de la etiología de la enfermedad que origine la IRA, por tratarse de cuidados específicos y generales en Nefrología. En consecuencia, parece que en casos de IRA puede ser pertinente el diseño y uso de la vía clínica.

Sin embargo para el caso clínico que se presenta, cobra importancia el registro de verificación de la vía clínica por recoger las adaptaciones a la evolución individual del proceso. Se ha tratado de planificar el máximo número de actividades, con objeto de evitar complicaciones y garantizar los procedimientos adecuados y su calidad.

El procedimiento seguido en hospitalización convencional se ajusta al protocolo de actuación de pacientes ingresados que precisan H.D. y que, al seguir una evolución similar pueden ser susceptibles de incluirse en una vía clínica de las características de las que se presentan en este caso:

El perfil de pacientes sería:

- Agudos que precisa H.D. en principio, un tiempo determinado.

- Crónicos que hayan ingresado durante el curso de otra enfermedad y precise H.D. durante su ingreso.

- Pacientes con ERC avanzada reagudizada que precisen entrar ya en programa de H.D.

- Pacientes de Diálisis Peritoneal que precisen H.D. provisionalmente.

- Personas con trasplante renal y deterioro de la función renal. 
El proceso de cuidados se valora y evalúa utilizando los patrones funcionales de M. Gordon que se han adaptado perfectamente a la idea de realizar un registro útil, gráfico y fácil de manejar para las enfermeras de las dos unidades a pesar de las peculiaridades de cada una de las unidades y la evolución de los pacientes.

\section{Limitaciones}

Este procedimiento se complica en servicios de Nefrología donde no comparten plantilla de enfermeras la unidad de Agudos y hospitalización convencional, y en los casos de permanecer ingresado el paciente en otra unidad.

Se propone, el uso de herramientas que faciliten una comunicación oral y escrita fluida entre las/los enfermeras/os de las dos unidades consensuando registros y compartiendo información de los cuidados de cada procedimiento así como la evolución y cambios en los diagnósticos.

El caso clínico que se ha presentado puede ser un ejemplo de discusión clínica en unidades de Nefrología.

\section{BIBLIOGRAFÍA}

1. Francisco Javier Gaínza ,Fernando Liaño García. Actuación en el fracaso renal agudo.Guías SEN.Nefrología.Vol.27.supl.3.2007.

2. L.Hernando Avendaño,P.Aljama Grcía,M.Arias Rodríguez,C. Caramelo Díaz,J.Ejido de los Ríos,S.Lamas Peláez. Nefrología clínica.3a edición.Panamericana. 2008.

3. M. J. Molina Garrido, C. Guillén Ponce, M. Guirado-Risueño, C. Martínez y Sevila, A. Carrato Mena. Servicio de Oncología Médica. Hospital General Universitario de Elche. Alicante.Diagnóstico diferencial de las gammapatías monoclonales.An.Med.Interna(Madrid)v.23n.11Madrid 
nov.2006.

4. J.Arrieta, C.Fernández Rivera, E.Gonzalez Parra. J.L. Gorriz TeruelJ.A.Herrero Calvo, R.LópezMenchero, V.Pérez Bañasco.et al.Guías de acceso vascular en hemodiálisis.Guías S.E.N. Nefrología.Vol.20.Supl.1.2005.

5. Lorena Delle Vedove Rosales,Ana Domingo Maldonado,Lourdes Fuentes Hernandez,Ana Hernandez Vallés,Rocío Gutierrez Dorta,Elena Pou Fernandez.Plan de cuidados en un paciente sometido a una biopsia renal.Elaboración de un registro de enfermería para la realización de una biopsia renal. Comunicaciones Presentadas al XXXV Congreso Nacional SEDEN. 2010.

6. http://www.senefro.org

7. http://www.juntadeandalucia.es/servicioandaluzdesalud/principal/default.asp

\section{COMENTARIOS DE INTERÉS DEL PROCESO CLÍNICO.}

Los tres primeros dias desde su ingreso (18-19-20) CPM hace unos balances positivos, superiores a 2000cc que convierten en prioritaria la reposicion de líquidos y el control de la ologoanuria (diuresis menor de 200 cada 24 hrs).

Al cuarto día se inicia HD y se sigue el estudio de su insuficiencia renal (analíticas,biopsia).

Durante diez días del 18 al 25 CPM es tratado de una IRA (N.T.A.N.TI.A.) de etiología múltiple (legionella,antibiótico nefrotóxico) hasta obtener los resultados del pico monoclonal en que se inicia el estudio de posible MM. El diagnóstico se confirma a los dieciseis días del ingreso.

Se inicia el tratamiento del MM en diecinueve dias desde su ingreso.

Tras la mejoría de la función renal, quedará a cargo de hematología oncologica con controles periodicos de nefrología. 


\section{REFLEXIÓN SOBRE LA RELEVANCIA DEL CUIDADO.}

El diagnóstico del MM se hace en el curso del estudio de la IRA causada por una Necrosis Tubular durante un periodo de diecinueve días. Durante este tiempo, el tratamiento y diagnóstico del paciente se lleva a cabo por un equipo multidisciplinar (en la unidad de Agudos y planta de Nefrología) y los cuidados por enfermeras especializadas en Nefrología donde la información es una prioridad sobre cada una de las pruebas, tratamientos y cuidados, diagnósticos y pronósticos además de coordinar aquellos que se comparten entre las dos unidades asistenciales: dos biopsias renales, más de diez hemodiálisis, transfusiones, tratamientos i.v. , inserción de tres CVC. requieren la puesta en marcha de un plan de cuidados consensuado que recoja todos los aspectos del seguimiento del paciente.Este podria ser un ejemplo a valorar y en su caso mejorar.

\section{ANEXOS}

\section{Elementos de la vía clínica que se adaptan:}

- Matriz temporal.

- Procedimientos de actuación

- Registro de verificación de actividades

- Evaluación

\section{CONCEPTOS Y DEFINICIONES:}

\section{Insuficiencia renal aguda y Necrosis tubular aguda.}

Para el funcionamiento renal son necesarias tres premisas: una perfusión sanguínea adecuada,la integridad del parénquima renal y la permeabilidad de las vías excretoras. La alteración súbita de cualquiera de estos elementos puede ocasionar un deterioro de la función renal llamado fracaso,insuficiencia o fallo(falla en latinoamérica). Dependiendo del elemento funcional alterado, el FRA se etiquetará como parenquimatoso o intrínseco si la alteración radica en las estructuras renales,prerrenal si lo que falla es la perfusión renal y 
obstructivo o post-renal si el flujo urinario está interrumpido.

El fracaso renal agudo (FRA) es un síndrome clínico, secundario a múltiples etiologías, que se caracteriza por un deterioro brusco de las funciones renales y que altera la homeostasis del organismo. Cursa, con frecuencia, con un descenso de la diuresis y siempre con un aumento de la concentración de las productos nitrogenados en sangre.

La interrupción brusca de la filtración glomerular define el FRA que como hemos dicho,puede deberse a múltiples causas. Entre ellas, de las que afectan directamente al parénquima renal, la necrosis tubular aguda(NTA) se caracteriza por un daño tubular selectivo con nula o mínima afectación glomerular o intresticial.El término NTA no implica una forma de muerte concreta de las células tubulares por lo que tiende a sustituirse por daño tubular agudo(acute kidney injury o AKI en inglés).La NTA puede desencadenarse por hipoperfusión renal que causa isquemia renal, por nefrotóxicos o por obstrucción tubular por el depósito intratubular masivo de moléculas. Las cadenas ligeras producidas y filtradas en exceso en el mieloma múltiple(MM) pueden obstruir la luz tubular y además son tóxicas para las células tubulares $1-2$.

\section{Mieloma Múltiple}

El mieloma múltiple (MM) es una enfermedad que se engloba dentro de las gammapatías que son un grupo de enfermedades caracterizadas por la proliferación de las células que sintetizan las inmunoglobulinas; casi siempre, esto conduce a la aparición de un componente monoclonal; en estos casos se habla de gammapatía monoclonal (GM). No siempre se trata de procesos malignos, sino que en ocasiones pueden ser benignos y consiste en una proliferación atípica de las células plasmáticas. El diagnóstico se realiza a partir de la aparición de un pico monoclonal en sangre o en orina. Las células plasmáticas ayudan al cuerpo a combatir la enfermedad produciendo proteínas Ilamadas anticuerpos. En el mieloma múltiple, las células plasmáticas crecen 
fuera de control en la médula ósea y forman tumores en áreas de hueso sólido. La proliferación excesiva de estos tumores óseos pesadas libres se producen solamente en condiciones que sea más difícil para la médula producir plaquetas y glóbulos sanguíneos saludables .

En condiciones normales, las células plasmáticas sintetizan moléculas completas de inmunoglobulina y cadenas ligeras libres. Las cadenas pesadas libres se producen solamente en condiciones patológicas. La cantidad habitual de síntesis de cadenas ligeras por día es de $0.9 \mathrm{gr}$. Estas cadenas ligeras viajan por el torrente circulatorio $y$, debido a su bajo peso molecular, son rápidamente eliminadas por el riñón por medio de filtración glomerular y reabsorción tubular, donde son catabolizados, creando aminoácidos simples que se devuelven a la circulación. El 99\% de las cadenas ligeras filtradas por el riñón son metabolizadas por los túbulos proximales. Normalmente se excreta una cantidad mínima de cadenas ligeras en la orina. En casos de producción exagerada de cadenas ligeras o de cadenas ligeras anormales, como ocurre en las disproteinemias, los lisosomas son incapaces de metabolizar adecuadamente estas cadenas ligeras. En algunos pacientes con mieloma múltiple, la síntesis de cadenas ligeras puede llegar a $85 \mathrm{~g} / \mathrm{día}_{2}$.

Las aparición de un pico monoclonal en sangre o en orina, es un hallazgo que aparece con bastante frecuencia en la práctica clínica habitual. Son múltiples las patologías que pueden ser responsables de tal dato, y conseguir llegar al diagnóstico definitivo determinará que el tratamiento sea el adecuado y que se modifique el pronóstico del paciente.

\section{Criterios diagnósticos de MM:}

- Pico monoclonal en sangre $y / u$ orina

- Infiltración de la médula ósea por células plasmáticas o plasmacitoma

- Afectación de órganos ó tejidos relacionados entre ellos: insuficiencia renal $_{3}$. 
En el caso que presentamos, este hallazgo se produce en el curso del estudio de una IRA con antecedentes de posible nefrotoxicidad por antibióticos y/o tratamiento de una legionela como causa de la NTA diagnosticada.

\section{RECOMENDACIONES SOBRE PROCEDIMIENTOS}

* Inserción cateter femoral: recomendaciones de la SEN (Sociedad Española de Nefrología): recomienda el uso de catéteres venosos centrales (CVC) en caso de previsión de una duración menor de 3-4 semanas . La enfermera preparará al paciente para la inserción de un CVC no tunelizado según la vena a canalizar,recto ó curvo, de 15 a $20 \mathrm{~cm}$ si es yugular y más de 20 si es femoral. Tras su uso en HD los cuidados y vigilancia seguirán las recomendaciones habituales de las Guías 4 .

* Primera HD: se preparará el monitor para una HD estandar según el protocolo del servicio, de corta duración y bajos flujos en la unidad de Agudos.

La HD es uno de los tratamientos sustitutivos de la función renal junto a la diálisis peritoneal(D.P.) y el trasplante renall que se realiza através de un acceso vascular por el que se pone en contacto la sangre del paciente con una solución através de una membrana semipermeable.Este tratamiento se utiliza para eliminar las toxinas urémicas y el exceso de líquidos del organismo(U.F.R. Ultrafiltración).

* Biopsia renal: El día antes de la biopsia se canalizará una vía periférica y se informará al paciente de que debe estaar en ayunas desde las 24 horas. Deberemos asegurarnos que está bien informado y en caso de precisarlo administrar medicación para dormir. Tras la realización de la técnica debemos estar alerta ante signos y síntomas de sangrado . Vigilaremos color y aspecto de la diuresis, tensión arterial, extracción y valoración de hemograma y reposo en cama hasta el día siguiente.

* Cuidados del CVC: En planta se vigilarán signos de sangrado y se evitará su uso para administrar medicación. Su uso será exclusivo para el personal de agudos que conectará al paciente a H.D. con asepsia y lo heparinizará según 
protocolos que se elaboran con las recomendaciones de la S.E.N.

\section{Fuentes bibliograficas utilizadas para realizar los procedimientos}

\begin{tabular}{|l|l|l|}
\hline Técnica/Cuidados & Actuación de enfermeria. & Fuente. Guias \\
\hline Insercion cateter femoral & $\begin{array}{l}\text { Preparacion de paciente y material segun } \\
\text { protocolo de la unidad. }\end{array}$ & $\begin{array}{l}\text { Guías s.e.n. accesos vasculares } \\
\text { año } 2004_{4}\end{array}$ \\
\hline Hemodialisis & Realizacion de 1á sesion de h.d de la unidad. & $\begin{array}{l}\text { Pauta individualizada del paciente } \\
\text { Protocolo de la unidad }\end{array}$ \\
\hline Biopsia renal & $\begin{array}{l}\text { Actuacion pre y post-biopsia } \\
\text { ayunas desde las 24 hrs. vía periférica. }\end{array}$ & $\begin{array}{l}\text { Plan de cuidados S.E.D.E.N. } \\
\text { año 20105 }\end{array}$ \\
\hline Mantenimiento vias centrales. & $\begin{array}{l}\text { Conexion- desconexion h.d. } \\
\text { Cuidados generales en planta } \\
\text { Administracion de medicacion por el c.v.c. }\end{array}$ & $\begin{array}{l}\text { Guías S.E.N. accesos vasculares } \\
\text { año 2004 }\end{array}$ \\
\hline
\end{tabular}

